\title{
Gib und Nimm.
}

\section{Lokale Austauschnetzwerke zwischen sozialer Bewegung und Marktergänzung}

\author{
Thomas Hinz und Simone Wagner ${ }^{1}$
}

\author{
Universität Konstanz
}

November 2005

\footnotetext{
1 Der Beitrag entstand im Rahmen des DFG-Projekts „Lokale Austauschnetzwerke“ (Hi 680/3-1). Kontaktpersonen: Dipl. Soz. Simone Wagner und Prof. Dr. Thomas Hinz, Universität Konstanz, D 78457 Konstanz. Die Autoren danken Werner Georg, Jochen Groß, Aja Tauschinsky und Herbert Kalthoff für Hinweise und Anregungen.
} 
Die Entwicklung des Kapitalismus wird seit seinen Anfängen von scharfer Kritik und Vorschlägen zu seiner Überwindung begleitet. Neben den politischen Fragen zur Eigentumsordnung, zur Rolle des Staates und zu Verteilungswirkungen des Marktes stand immer auch die Funktion der Geldwirtschaft im Zentrum der Aufmerksamkeit. Warum sollen Menschen nicht bedarfsgerecht wirtschaften - ohne sich den Restriktionen knappen Geldes auszusetzen? Jede und jeder soll nach ihren und seinen Fähigkeiten und Bedürfnissen an der Gesellschaft und damit auch an wirtschaftlichem Austausch teilnehmen können, so lautet eine bekannte Forderung zur Überwindung der kapitalistischen Geldwirtschaft. Die daraus abgeleitete Idee von Tauschringen und einer Alternativwährung formierte sich bereits im 19. und frühen 20. Jahrhundert als soziale Bewegung zur Überwindung wirtschaftlicher Fehlentwicklungen. Deutschland wurde in den 1990er Jahren von einer neuen Tauschringbewegung erfasst, die sich schnell ausbreitete. Während 1995 knapp 60 Tauschringe in Deutschland existierten, zählte man Ende 1996 bereits 114 und 2002 etwa 350 Zusammenschlüsse (Meier 2001; Täubner 2002). Die Tauschringe wollen bei ihren Mitgliedern die Möglichkeiten zur Selbsthilfe stärken und dadurch deren wirtschaftliche Situation verbessern (Williams 1996a; 1998), gleichzeitig besitzen sie auch heute einen sozialutopischen Anspruch gegen die Globalisierung und ihre negativen Folgen (Gran 1998): Zumindest im regionalen Format wird eine sozial gerechtere Welt angestrebt. Diese Zielsetzungen sollen innerhalb eigener alternativer Wirtschaftskreisläufe verwirklicht werden, in die Teilnehmer ihre Ressourcen und Fähigkeiten einbringen und austauschen (Williams 1995; 1996b). Regionale Alternativwährungen sollen helfen, die Abhängigkeit von der nationalen Währung, deren Verfügbarkeit häufig an Erwerbseinkommen gebunden ist, aufzubrechen und den Teilnehmern einen bedürfnisgerechten Austausch ermöglichen (Hoeben 2003; Pacione 1998, S. 65; Williams 1997).

Unser Beitrag widmet sich der Entstehung, Verbreitung und Bestandssicherung von Tauschringen seit den 1990er Jahren und führt einen möglichen Analyserahmen für die Untersuchung dieser Fragestellungen ein. Bei der Betrachtung des Gründungs- und Diffusionsprozesses der Tauschringbewegung wird eine populationsökologische Herangehensweise vorgeschlagen. Diese stellt die Bedeutung von Umweltbedingungen für den Gründungsprozess heraus (Stinchcombe 1965) und beleuchtet die Relevanz von Ideologien zur Durchsetzung neuer Organisationsformen (Rao 2002). Dabei spielen auch die politischen Entrepreneurs eine besondere Rolle (Kimberly 1980; Olson 1968). Auch zur Analyse der Bestandssicherung wird eine populationsökologische Rahmung entworfen, da mit ihrer Hilfe Faktoren, die auf den Fortbestand der Organisationen Einfluss nehmen, herausgearbeitet werden können. Außerdem werden an dieser Stelle die Konzepte der sozialen Einbettung und des Sozialkapitals als mögliche Instrumente zur Untersuchung der einzelnen Transaktionen gewählt (Granovetter 1985; 1990; Coleman 1988; 1990). Denn die soziale Einbettung bietet ins- 
besondere in problematischen Austauschbeziehungen eine sinnvolle Ergänzung zur vertraglichen Absicherung (Voss 1998). Die Argumentation zeigt mögliche theoretische Annäherungen für die behandelten Fragestellungen auf. ${ }^{2}$ Die tatsächliche Anwendbarkeit dieser Ansätze auf die Tauschringthematik muss an anderer Stelle mit Hilfe empirischer Daten überprüft werden.

Der Beitrag gliedert sich in fünf Abschnitte. Zunächst geht es darum, die Geschichte der Tauschringe und deren Funktionsweise knapp zu beschreiben (Abschnitt 1). Im Anschluss beschäftigen wir uns mit Faktoren, welche die Entstehung und Verbreitung der Tauschringe beeinflussen können (Abschnitt 2). Auch die Stabilisierung der Tauscharrangements ist von fundamentalem Interesse (Abschnitt 3). Schließlich widmen wir uns den in der Einleitung angesprochenen Ansprüchen und Möglichkeiten der Tauschringe: Sind sie als Marktalternative und Sozialutopie tragfähig, wie einige Personen der Tauschringbewegung behaupten (Abschnitt 4)? In der Zusammenfassung diskutieren wir, was die Soziologie aus der theoriegeleiteten und empirischen Auseinandersetzung mit Tauschringen lernen kann (Abschnitt 5).

\section{Tauschringe - Geschichte und Funktionsweise}

Komplementäre Wirtschaftssysteme sind keine neue Erfindung. Bereits im 18. Jahrhundert bildeten sich erste Tauschsysteme heraus. Diese Initiativen verfolgten sozialreformerische Absichten und begriffen sich vornehmlich als Reaktionen auf den sich entwickelnden Kapitalismus. Die nicht-monetären Austausch- und Versorgungssysteme wie beispielsweise die Arbeitszeitbörsen von Robert Owen oder die Tauschbanken von Joseph Pierre Proudhon sollten helfen, die mit der kapitalistischen Entwicklung einher gehende ungleiche Verteilung (die „Einkommensschere“ zwischen Arm und Reich geht immer weiter auseinander) zu korrigieren. $^{3}$

Größere Popularität erlangten die Tauschringe in der Weltwirtschaftskrise um 1930. Viele Projekte aus dieser Zeit bauen auf der Freigeldtheorie des Finanztheoretikers Silvio Gesell (1862-1930) auf. Laut Gesell sind die bestehende Geldordnung und die Wertaufbewahrungsfunktion des Geldes für die Fehlentwicklung der Wirtschaft verantwortlich. Denn die Wertaufbewahrungsfunktion verleitet zur Hortung von Geldbeständen und führt dazu, dass Geld dem Wirtschaftskreislauf entzogen wird. Zur Lösung dieses Problems schlug er das

\footnotetext{
${ }^{2}$ An Hand der Tauschsysteme könnten auch weitere Fragestellungen diskutiert werden, die im Zuge einer „Neuen Wirtschaftssoziologie“ eine Rolle spielen (Smelser/Swedberg 2005). So eröffnen sich die Tauschringe beispielsweise einer interpretativ-kultursoziologischen Betrachtungsweise, die insbesondere an der sozialen Bedeutung der Alternativwährung ansetzen kann (Zelizer 1994).

${ }^{3}$ Die Ursache für die ungleiche Einkommensentwicklung liegt im System selbst: Die Kapitalisten können unter Berufung auf Eigentumstitel die Gewinne einbehalten. Miet-, Pacht- und Darlehensverträge sichern ihnen zusammen mit dem Zinsmechanismus weitere Einkommensquellen (Binn 1982; Meier 2001).
} 
Freigeld vor - ein sich periodisch selbst entwertendes Papiergeld - das als „Objekt des Hortens“ uninteressant werden sollte (Offe/Heinze 1990). Insbesondere das Nothilfeprogramm der Tiroler Gemeinde Wörgl ist in diesem Zusammenhang hervorzuheben. Gesells Freigeld findet sich in diesem Experiment in Form der Arbeitsbestätigungsscheine wieder. Mit deren Hilfe sollten für die Gemeindemitglieder Arbeitsmöglichkeiten geschaffen, der Warenumsatz gehoben und die ausständige Steuer eingebracht werden (Unterguggenberger 1983). Trotz nachweisbarer Erfolge wurde das Projekt nach etwa einjähriger Laufzeit eingestellt, da die Österreichische Nationalbank ihr Banknotenprivileg verletzt sah (Hubert 2004; Onken 1983). Unterstützt durch die Militärregierung wurden auch im Nachkriegsdeutschland abgewandelte Formen von Tauschringen initiiert, um der Ausbreitung von Schwarzmärkten und der schlechten Versorgungslage der Bevölkerung mit zusätzlichem Angebot zu begegnen. ${ }^{4}$

Die angesprochene Renaissance erleben die Tauschringe in Form von Local Exchange Trading Systems (Godschalk 1986; Kuhn 2002; Paysys 1997). Das erste LET-System wurde 1983 in einer kleinen Stadt in British Columbia, Kanada, als Reaktion auf eine ökonomische Depression von Michael Linton gegründet (Hoeben 2002; Pacione 1998; Williams 1995). ${ }^{5}$ Mit seinem Projekt sollte die aufgrund hoher Arbeitslosigkeit lahmende lokale Wirtschaft wieder angekurbelt werden. Linton baute sein Tauschnetzwerk in Anlehnung an die kommerziellen Barter Clubs zu einem lokalen Währungssystem aus, welches indirekten und multilateralen Austausch ermöglichte (Meier 2001; Peterson 1990). Innerhalb des Systems geleistete Arbeiten werden mit einer eigenen Parallelwährung entlohnt, so dass auch Personen, die nicht über ausreichende Geldbestände verfügen, im alternativen Wirtschaftskreislauf tätig sein und die durch Arbeitslosigkeit verursachten, wirtschaftlichen Einbußen ein Stück weit ausgleichen können (Caldwell 2000; Maier 2001; North 1999; Williams 1996a). Leistungen außerhalb des Systems können mit diesen Guthaben jedoch nicht erworben werden, da die Verrechnungseinheiten nicht in die Landeswährung konvertierbar sind (Hubert 2004). Allerdings war die ursprüngliche Alternativwährung an die Landeswährung gekoppelt, so dass ein Umrechnungskurs gegeben war. Diese Kompatibilität ermöglicht den Handel zwischen, als auch die unternehmerische Beteiligung an diesen Tauschsystemen. Allerdings spiegeln sich bei dieser „Bezahlungsform“ die Bewertungen des Marktes wider. Die Preise werden unter den Teilnehmern in Anlehnung an die Marktpreise ausgehandelt. Die am Markt bestehende Ungleichheit zwischen qualifizierten und unqualifizierten Arbeitnehmern sowie zwischen Männern und Frauen wird somit reproduziert (Bowring 1998;

\footnotetext{
${ }^{4}$ Hierbei handelt es sich beispielsweise um Tauschzentralen - etwa in München-Haidhausen. Entgegen bisher vorgestellter Formen und moderner Tauschsysteme handelt es sich bei dieser Erscheinung um ein duales Währungssystem in dem zwei Tauschmittel gleichberechtigt nebeneinander bestehen (Hubert 2004; Schneider 1995) An- und Verkauf erfolgte je nach Verfügbarkeit mit Geld oder Tauschbons.

${ }^{5}$ Es handelt sich um die Stadt Courtaney in Comox Valley (Pacione 1997).
} 
Lang 1994; Seyfang 2002). Um den sozialutopischen Zielen näher zu kommen, greifen die meisten neueren Systeme daher auf zeitbasierte Zahlungsmittel zurück. In diesen Arrangements wird eine Stunde Arbeit unabhängig vom Marktwert der Leistung gleich bewertet und entlohnt (Bebbington 2000; Lee 1996; Thorne 1996). Geld existiert in diesen Tauschsystemen nur im „virtuellen Sinne“, der Austausch erfolgt „bargeldlos“ über Gutschriften auf den Konten der am Tausch beteiligten Personen (Bebbington 2000; Pieper 2002).

Das Tauschgeschehen kann man sich in etwa folgendermaßen vorstellen: Teilnehmer A fragt bei Teilnehmer B eine Dienstleistungsstunde, etwa Bügeln, nach. Während Teilnehmer B eine Gutschrift über diese Leistung auf seinem Konto erhält, verringert sich der Kontostand des Teilnehmers A um diesen Betrag. Da auf eine Soll- und Habensverzinsung sowie eine Kreditobergrenze verzichtet wird, können auch Teilnehmer mit negativen Kontoständen Dienste und Waren in Anspruch nehmen, ohne zuvor ihren Kontostand wieder durch eigene Leistung auszugleichen. Die an Gesells Freigeldtheorie angelehnten Vorstellungen zielen darauf ab, der Hortung von Geldbeständen vorzubeugen und die Zirkulation des „Kapitals“ innerhalb des Systems zu gewährleisten. Die Geldmenge wird nicht zentral von der Tauschzentrale emittiert, sondern von den beteiligten Individuen eigenverantwortlich koordiniert. Sie wird also über die Nachfrage und Tauschvorgänge der einzelnen Teilnehmer geschöpft (Meier 2001; Pacione 1997; 1998). Linton begreift seine Tauscharena als ein „self-regulating economic network which allows its members to issue and manage their own money supply within a bounded system” (Ekins 1986). Zum Schutz des Systems vor Missbrauch besteht innerhalb der lokalen Austauschnetzwerke allerdings kein „Bankgeheimnis“, jeder Teilnehmer hat Einblick in die Kontostände, Transaktionsvolumina und Umsätze seiner Tauschpartner (Caldwell 2000; Carmen 1997; Meier 2001; Schraven 2000; 2001). ${ }^{6}$

Lintons LET-System wurde von seinen Teilnehmern sehr gut angenommen und erhielt regen Mitgliederzuwachs. Schon bald nach seiner Einführung verbreitete sich die Idee im gesamten englischsprachigen Raum und in den frühen 1990er Jahren erfasste sie schließlich auch Deutschland. Abgesehen von regionalen Variationen in Bezug auf Währung, Handelsvolumen, Mitgliedsprofil und gemeinschaftlichen Veranstaltungen sind diese Systeme alle sehr ähnlich aufgebaut und eng an die Struktur ihres kanadischen Vorbilds angelehnt (Pacione 1998). ${ }^{7}$

\footnotetext{
${ }^{6}$ Durch diese Ausgestaltung besteht die Gefahr, dass Trittbrettfahren zur individuell rationalen Strategie wird. Denn es werden Anreize gesetzt, dass Teilnehmer nur nehmen und nicht geben und den Tauschring dann wieder verlassen.

${ }^{7}$ Alle Systeme arbeiten mit lokalen, nicht in die Landeswährung konvertierbaren Tauschmitteln, distanzieren sich von einer Soll- und Habensverzinsung und einer zentralen Geldmengensteuerung.
} 


\section{Tauschringe als Organisationsform}

Tauschringe als Organisationsform sind Ausdruck einer sozialen Bewegung, für deren Entstehen die Bedeutung von politischen Entrepreneurs hervorzuheben ist. Sie spielen mit intrinsischen Motivationen für das Zustandekommen der Organisationsform und den Aufbau kollektiver Identitäten eine bedeutsame Rolle (Whittier 1997). Die Entstehung der lokalen Austauschnetzwerke ist - so zeigen bisher durchgeführte Studien im englischsprachigen Raum - zu einem wichtigen Teil auf das Engagement einzelner, ökologisch motivierter Personen zurückzuführen. Die Initiatoren verfolgen mit ihren als Protestbewegung zur Mainstream Ökonomie gegründeten Systemen häufig ideologische Zielsetzungen (Gran 1998). Außerdem erfordert der Kollektivgutcharakter von sozialen Bewegungen von den politischen Entrepreneurs Vorleistungen in Form von finanziellem, sozialem und kulturellem Kapital. ${ }^{8}$ Gerade im frühen Stadium einer sozialen Bewegung müssen Ressourcen mobilisiert, koordiniert und zusammengeführt werden und die neue Bewegung muss durch die gewählte Organisationsform politische und konstitutive Legitimität erlangen (Anheier 2000; Rao 2002). Politische Legitimität erreicht die neue Organisationsform durch die Einhaltung rechtlicher Vorschriften und indem sie Unterstützung mächtiger Akteure gewinnt, konstitutive Legitimität hat sie dagegen erlangt, sobald sie als selbstverständliche „social fact“ betrachtet wird (Hannan/Caroll 1992). Auch in diesem Zusammenhang wird die Bedeutung politischer Entrepreneurs für die Durchsetzung neuer Organisationsformen herausgestellt. Dadurch dass politische Entrepreneurs häufig im politischen Umfeld aktiv sind, die Unterstützung einflussreicher Dritter gewinnen und entscheidungsrelevante Informationen erhalten und verbreiten, tragen sie wesentlich zur Legitimität einer neuen Organisationsform bei, die aus einer sozialen Bewegung hervorgegangen ist. In einer Kurzdarstellung zum Stand der Forschung zu sozialen Bewegungen zeigen McAdam et al. (1996), dass die Möglichkeiten, Veränderungen auch durchzusetzen, in entscheidendem Umfang von den Mobilisierungsstrukturen und politischen Gelegenheiten der politischen Entrepreneurs abhängen. Anheier (2000) geht weiterhin auf die Bedeutung politischer Entrepreneurs für die gezielte Weitergabe von Informationen bei der Entstehung einer neuen politischen Gruppierung ein (vgl. auch Rao 2002). ${ }^{9}$ Sehr wahrscheinlich sind auch die lokalen Austauschnetzwerke bei der Ressourcenmobilisierung und Erschließung von Legitimitätsquellen auf die Aktivität eines politischen Entrepreneurs angewiesen. So zeigen Studien zum Teilnehmerprofil von Tauschsystemen des englischsprachigen

\footnotetext{
${ }^{8}$ Da hiermit nicht unbedingt zu rechnen ist und nicht auf die Opferbereitschaft von Privatpersonen gezählt werden kann, empfehlen die „Mentoren“ der Bewegung, Claus Offe und Rolf Heinze, dass stützende, fördernde Initiativen von Landes- oder Kommunalbehörden die Entstehung von Tauschringen vorantreiben sollten (1990, S. 92).

${ }^{9}$ Seine Studie befasst sich mit der Verbreitung der NSDAP in Deutschland.
} 
Raums, dass die Initiatoren dieser Austauschnetzwerke häufig grün-alternativen Bewegungen, Organisationen oder Parteien nahe stehen (Gran 1998; Langseth 1993; Seyfang 1997).

Für neue Organisationsformen ist es außerdem bedeutsam, ihre Interessen, Ziele und Aufgaben ihren Teilnehmern und der Organisationsumwelt zu vermitteln. Nur wenn dies erfolgreich geschieht, können zusätzliche Teilnehmer und Unterstützer gewonnen werden. Dabei helfen auch durch die politischen Entrepreneurs geschaffene „kollektive Identitäten“ (Aldrich 1999; Kimberly 1980; Rao 2002). Bei den Austauschnetzwerken sind Vorstellungen über die gemeinsamen Ziele und die Strategien, sie zu erreichen, von zentraler Bedeutung für die weitere Entwicklung. Einige Studien zeigen, dass sich in Tauschsystemen im Zeitverlauf ein „othering“ entwickelt, und Personen, die mit ihrer Teilnahme andere Ziele verfolgen, informell von diesen Zusammenschlüssen ausgeschlossen werden. Auf eine Abgrenzungsstrategie deutet zugleich der Weg zur Anwerbung neuer Mitglieder hin. Neue Mitglieder werden fast ausschließlich durch gezielte Ansprache interessierter Organisationen - vor allem Umweltorganisationen - oder durch Mund-zu-Mund-Propaganda gewonnen. Die stark ideologische Ausrichtung der Tauschringe und ihre selektive Mitgliedergewinnung sind als auf Identitätsbildung abzielende Handlungen von politischen Entrepreneurs interpretierbar (Seyfang 1997; Williams 1996b; 1997; 1998).

Welche Faktoren werden außer der Etablierung der Organisationsform bei der Gründung von Organisationen wirksam? Mit dieser Frage wird eine weitere Tradition der Organisationsforschung aufgegriffen, die sich auf Stinchcombes Aufsatz „Organizations and Social Structure“ (Stinchcombe 1965) stützt und sich mit der Beziehung zwischen Organisationen und ihrer Umwelt auseinandersetzt. Häufig führt erst das Zusammenspiel einer Reihe von Umweltbedingungen zur Idee bzw. Entscheidung, eine neue Organisation zu gründen und die Gründungsumstände prägen die Organisationen mit langfristiger Wirkung (Kimberly 1980, S. 26). Damit richtet sich der Fokus nun auf organisationale Attribute und Umweltbedingungen und ihre Konsequenzen für den Erfolg der Gründungsversuche (Romanelli 1989). Zahlreiche Studien arbeiten Faktoren heraus, die Einfluss auf die Gründungsaktivität nehmen können. Wesentlich ist, dass die neuen Organisationen Zugang zu gründungsrelevanten Ressourcen haben. Hierzu zählen ökonomische Ressourcen in Form von Ersparnissen oder Venture Capital und soziale Ressourcen wie beispielsweise Netzwerkbeziehungen oder qualifiziertes Personal (Aldrich/Pfeffer 1976; Pennings 1980, S. 144f). Aufgrund der Nähe der Tauschringe zur informellen Ökonomie kann auch die sozial- und steuerrechtliche Toleranz der beheimatenden Region den Gründungsprozess beeinflussen.

Weiterhin regen erst die Bedingungen und Veränderungen der formellen Ökonomie die Initiatoren und Teilnehmer zur Betätigung in der informellen Ökonomie an. Diese Annahme knüpft an die in der Organisationsforschung weit verbreitete Nischentheorie an. Für die Gründung neuer Organisationen nehmen ungenutzte Nischen eine herausragende Be- 
deutung ein. Eine Nische „consists of the social, economic, and political conditions that can sustain the functioning of organizations that embody a particular form” (Hannan/Carroll 1995, S. 34). Laut Pennings (1980) bieten Nischen neuen Organisationen besonderes Potenzial, da sie sich bisher frei verfügbare Ressourcen aneignen und dadurch gegenüber der Konkurrenz, insbesondere aber gegenüber späteren Imitatoren, Vorteile - so genannte „first-moveradvantages“ verschaffen können. Häufig ergeben sich solche „ecological opportunities“ aufgrund veränderter Umweltbedingungen. So können beispielsweise technologischer Wandel, veränderte institutionelle Rahmenbedingungen, ökonomische Schwankungen aber auch politische Umstürze, Entwicklungsmöglichkeiten für neue Organisationsformen bieten (Astley 1985; Brittain/Freeman 1980, S. 294f; Pennings 1980, S. 138; Romanelli 1989; Stanley 1981, S. 96; Dobrev et al. 2001). Im Fall der Austauschnetzwerke spricht für diese These der bereits ausgeführte Befund, wonach die Tauschsysteme in Reaktion auf ökonomische Krisen entstanden (Hoeben 2003; Seyfang 2002; Williams 1997). Außerdem werden in der Forschung für die Ausbreitung der informellen Ökonomie häufig Aspekte wie Staatsversagen, Marktversagen, strukturelle Veränderungen am Arbeitsmarkt und Wertewandel als Hauptursachen genannt (Schneider/Enste 2000).

Ein weiteres wesentliches Merkmal im Zusammenhang mit Organisationsgründungen stellt die Dichteabhängigkeit von Neugründungen dar. Da die neue Organisationsform zu Beginn erst soziale Akzeptanz erlangen muss, existieren in der frühen Entwicklungsphase häufig nur wenige Populationsmitglieder. Mit zunehmender sozialer Anerkennung der neuen Organisationsform steigt jedoch auch die Anzahl neuer Mitglieder in der Population. Allerdings geht mit dieser Ausbreitung auch verstärkter Wettbewerb für die Population einher, so dass das Wachstum allmählich durch zunehmende Sättigungstendenzen eingeschränkt wird. Somit werden bei Diffusionsprozessen neuer Organisationsformen zwei gegenläufige Prozesse wirksam: Legitimation und Wettbewerb (Hannan/Carroll 1992; Baum 1996). Wahrscheinlich verläuft auch der Diffusionsprozess der Tauschringe entsprechend der These der Dichteabhängigkeit und es ist zu erwarten, dass auch Tauschringe nicht in unbegrenztem Umfang neu geschaffen werden. Es spricht eine deskriptive Studie deutscher Tauschsysteme (Niemeyer 2002), die sich auf die Datengrundlage des von Klaus Kleffmann gepflegten Tauschringarchivs stützt, dafür, dass Sättigungsgrenzen erreicht werden. Nach den ersten Gründungen in 1992 erreichte die Welle an Neugründungen zwischen 1995 und 1996 ihre Spitze und ebbte dann nach und nach ab. ${ }^{10}$

\footnotetext{
${ }^{10}$ Diese Studie spiegelt jedoch nur einen groben Trend der deutschen Tauschringlandschaft wider, da in diesem Archiv nur Tauschringe enthalten sind, die sich dort eigeninitiativ registrierten.
} 


\section{Stabilisierung der Tauschnetzwerke}

Nach der Gründungsphase müssen neue Organisationen vielfältige, sich teilweise widersprechende Herausforderungen meistern, um sich langfristig zu etablieren. So gilt es, die mit der Gründung zum Ausdruck kommende Ideologie zu bewahren und gegebenenfalls auch weiter zu entwickeln, gleichzeitig muss Effizienzkriterien gerecht geworden werden (Miles 1980; S. 436). Auch Tauschringe stehen in diesem Spannungsfeld. Zwar wird ihre Leistungsfähigkeit nicht nur nach der Dimension ökonomischer Effizienz bemessen, aber dennoch sollten sie klassische Bestandsprobleme lösen (Miles 1980). Sie müssen eine ausreichende Tauschaktivität und Angebotsdiversifikation herstellen (technical), ihre Mitglieder halten und neue Teilnehmer hinzugewinnen (political) und sich eine ideologische Ausrichtung aufbauen und bewahren (cultural). Im Hinblick auf die Beiträge der individuellen Teilnehmer werden die Austauschnetzwerke außerdem nur überleben, so lange die Anreize einer Teilnahme die Kosten der Teilnahme überwiegen (March/Simon 1958).

Bereits in der Gründungsphase werden für neue Organisationen wesentliche Weichen im Hinblick auf ihr weiteres Fortbestehen gestellt. Zu diesem frühen Zeitpunkt werden wichtige Struktureigenschaften der Organisation selbst festgelegt, welche die Überlebenswahrscheinlichkeit der Gründung beeinflussen (Stinchcombe 1965). Daher dürfte auch der Erfolg eines Tauschrings zumindest teilweise auf frühe Festlegungen im Gründungsprozess zurückzuführen sein. Außerdem hängt die Überlebenswahrscheinlichkeit neuer Organisationen von den Eigenschaften des politischen Entrepreneurs ${ }^{11}$ sowie den Umweltbedingungen ${ }^{12} \mathrm{ab}$. Neben diesen bereits bei Organisationsgründung wirksam werdenden Einflüssen spielen auch Größe und Alter der Organisation eine wichtige Rolle für ihre Überlebenschancen. Der Überlebensverlauf von Tauschringen dürfte gemäß der „liability of adolescence“ verlaufen. ${ }^{13}$ Nach anfänglicher Schonfrist werden diejenigen Tauschringe, die ihre meist euphorischen, ideologisch behafteten Zielsetzungen weit verfehlen, die notwendige Mindestgröße nicht erreichen oder durch free riding in eine „Schuldenfalle“ geraten, wieder eingestellt. Dafür

\footnotetext{
${ }^{11}$ Dazu zählen Kapital- und Humankapitalausstattung, aber auch Zugang zu relevanten Netzwerken und die ideologische Ausrichtung (Brüderl/Preisendörfer/Ziegler 1992; Hinz 1998; Pennings 1980).

${ }^{12}$ Beispielsweise Marktkonzentration, politisches Klima, gesamtwirtschaftliche Lage (Brittain/Freeman 1980; Carroll 1983; Hinz 1998).

${ }^{13}$ Gemäß der These der „liabilities of newness“ unterliegen neu gegründete Organisationen einem deutlich höheren Risiko zu scheitern. Dies liegt mitunter darin begründet, dass neuere Organisationen noch keine „stabilen Verbindungen zu Konsumenten und Kunden der von den Organisationen angebotenen Dienste“ aufbauen konnten (Freeman/Hannan/Carroll 1984; Stinchcombe 1965). Die These der „liabilities of adolescence“ spricht sich dagegen dafür aus, dass eher etwas ältere Organisationen einer höheren Sterblichkeit ausgesetzt sind, die ihren anfänglichen Vertrauensvorschuss quasi „verspielt“ haben. Erst nach bestandener „Reifungskrise“ geht ihr zu Folge die Hazardrate wieder zurück (Fichman/Levinthal 1991). Beim Einfluss der Organisationsgröße auf das Sterberisiko wird meist die These der „liabilities of smallness“ vertreten. Aufgrund von Wettbewerbsnachteilen und geringerer Ressourcenausstattung zu Beginn der Geschäftstätigkeit unterliegen kleinere Unternehmen demnach einem vergleichsweise höheren Risiko zu scheitern (Aldrich/Auster 1986; Brüderl/Preisendörfer/Ziegler 1992; Hinz 1998).
} 
spricht, dass häufig nur verhältnismäßig junge und relativ alte Tauschringe bestehen. Außerdem dürften kleinere Austauschnetzwerke einer höheren Gefahr des Scheiterns ausgesetzt sein, da sie aufgrund ihrer geringeren Teilnehmerzahlen ein weniger differenziertes Angebot vorweisen können und daher viele Wünsche innerhalb dieser Arrangements nicht befriedigt werden können (Williams 1997).

Der Fokus lag bisher auf Tauschringen als Organisationsform sowie der gesamten Population von Tauschringen. Die Art und Weise, wie Tauschsysteme angelegt sind, kann aber auch auf der Mikroebene - also auf Ebene der einzelnen Transaktionen - zu Bestandsproblemen führen: Opportunismus und Trittbrettfahrerverhalten. Informationsasymmetrien zwischen Aufraggeber und Auftragnehmer eröffnen einen Spielraum für opportunistische Verhaltensweisen - also für Handlungen, die den eigenen Nutzen auf Kosten des Partners mehren (Williamson 1985). In entsprechenden Beziehungen versucht der Auftraggeber häufig, den Auftragnehmer mit geeigneten (meist monetären) Anreizen zu motivieren, Entscheidungen im Sinne des Auftraggebers zu treffen (Kleine 1995; Picot 1999). Diese Möglichkeit, solche „Agenturprobleme“ abzufedern, kann im Zusammenhang mit den lokalen Austauschnetzwerken nicht herangezogen werden, da aufgrund der meist verwendeten Zeitwährung eine Preisdifferenzierung nicht möglich ist. Abgesehen von den fehlenden marktwirtschaftlichmonetären Anreizen wird die Tendenz des Opportunismus und free riding zusätzlich verschärft, da von Zinsmechanismen, Kreditobergrenzen und vertraglichen Absicherungen abgesehen wird. Das Prinzip der Austauschnetzwerke begünstigt somit eine typische Kollektivgutsituation: Individuell rationales, Nutzen maximierendes Verhalten führt zu sozial ineffizienten Ausgängen, etwa zum Zusammenbruch des Systems aufgrund von „Überschuldung““ ${ }^{14}$ Welche Absicherungsmechanismen können in einem solchen Umfeld genutzt werden, um einen reibungslosen Ablauf der Tauschgeschäfte zu erzielen und ausbeuterischen Aktionen vorzubeugen und somit auch den langfristigen Bestand des Systems zu ermöglichen?

Bei den lokalen Austauschnetzwerken dürfte vor allem Vertrauen unter den Teilnehmern eine bedeutsame Stellung einnehmen. Doch wie entsteht Vertrauen? ${ }^{15}$ Eine sinnvolle Ergänzung zur vertraglichen Absicherung, die im Fall der Tauschringe als wirksames Mittel nicht zur Verfügung steht, bietet die soziale Einbettung einer Beziehung (Abraham/Voss 1999; Macaulay 1963; Voss 1998). In problematischen Austauschbeziehungen kann soziale Einbindung Vertrauen schaffen und die Handlungsergebnisse der Akteure nachhaltig be-

\footnotetext{
${ }^{14}$ Beispielsweise kann sehr hohe Kontoüberziehung oder gar Austritt ohne Schuldenrückzahlung in dieser Umgebung zur individuell rationalen Strategie werden und eine kollektive Überschuldung des Systems mit einem katastrophalen Geldmengenüberangebot bewirken. Eine weitere individuell rationale Strategie kann das Angebot qualitativ minderwertiger Leistungen werden. Bei einer Ausbreitung dieser Strategie würde das System für die Teilnehmer langfristig uninteressant.

${ }^{15}$ Mit der Herstellung von Vertrauen befassen sich bereits verschiedene Studien zu Transaktionen, die über den Markt abgewickelt werden (Abraham/Kropp 2000; Batenburg/Raub/Snijders 2000; Raub/Weesie 1990). Auch im Markt reichen Verträge aufgrund zwingender Unvollständigkeiten häufig nicht aus, das Opportunismuspotenzial einzudämmen (MacNeil 1978).
} 
einflussen. Daher wird sie als Substitut für Vertragslösungen oder den Aufbau von interner Hierarchie herangezogen, sie gilt als eine Dimension von Sozialkapital (Flap 1988; Granovetter 1985; 1990, S. 95; Voss 1998). Die zeitliche Einbettung einer Transaktion resultiert aus einer gemeinsamen Vergangenheit als auch der Erwartung einer gemeinsamen $\mathrm{Zu}$ kunft. Beim „Schatten der Vergangenheit“ vermindern Lerneffekte über das Verhalten des Partners und wechselseitige beziehungsspezifische Investitionen die Wahrscheinlichkeit opportunistischer Verhaltensweisen (Raub 1999, S. 243; Snijders 1996). ${ }^{16}$ Beim „Schatten der Zukunft“ stabilisiert sich wechselseitiges Vertrauen durch bedingte Kooperation, so dass es individuell rational wird, der Reziprozitätsnorm zu folgen (Axelrod 1987; Batenburg/Raub/ Snijders 2000, S. 396; Raub 1999, S. 243). ${ }^{17}$ Die Versuchung, zu opportunistischem Verhalten kann außerdem durch strukturelle Einbettung der Transaktion eingedämmt werden. In andauernden Beziehungen können sich Reputationseffekte ausbilden, wenn Informationen über die Verhaltensweise eines Akteurs in einer seiner Beziehungen über Informationsnetzwerke zu seinen anderen Partnern gelangen. Daher muss ein Akteur bei opportunistischen Verhaltensweisen in einer solchen Konstellation zusätzlich mit Reaktionen dritter Parteien rechnen (Raub/Weesie 1990; Voss 1998).

Auch in den Austauschnetzwerken dürfte die soziale Einbettung zur Absicherung der Tauschgeschäfte wirksam werden. Dafür sprechen die von den Tauschringen regelmäßig veranstalteten Treffen, die den Teilnehmern ein gegenseitiges Kennenlernen und Austauschen ermöglichen und auf diese Weise das Zusammengehörigkeitsgefühl stärken sollen. ${ }^{18}$ Bei der Lösung des Ordnungsproblems spielt daher auch die Netzwerkstruktur eine entscheidende Rolle. Jedoch wirken sich bestimmte Netzwerkeigenschaften günstiger als andere aus (Haug 2000). Kleine Gruppen oder hohe Netzwerkdichte sind bei der Bereitstellung von Kollektivgütern großen und losen Zusammenschlüssen überlegen. Sie stellen unmittelbare Beobachtbarkeit und Sanktionierbarkeit her, da häufige, enge Beziehungen die unmittelbare Informationsweitergabe und somit die Entstehung von Reputationseffekten begünstigen. Die Akteure werden daher ihre persönlichen Motivationen aufgrund ihrer Nutzenerwartungen den Erwartungen der Gruppe unterordnen (Olson 1968; Coleman 1988; 1990). Wahrscheinlich werden auch in Tauschringen günstige Netzwerkeigenschaften opportunistischen Verhaltensweisen und ausbeuterischen Tendenzen gegensteuern. Denn meist handelt es sich bei diesen Zusammenschlüssen um relativ kleine Gruppierungen von 60 bis 100 Teilnehmern (Niemeyer 2002).

\footnotetext{
${ }^{16}$ Also Investitionen, welche die Beziehung für beide Seiten attraktiver gestalten und bei Abbruch einer Beziehung abgeschrieben werden müssen

${ }^{17}$ Die Erwartung einer Tit-for-Tat Strategie mit der Aussicht auf zukünftige Sanktionen wird die Versuchung verringern, opportunistischen Verhaltensweisen zu erliegen, sofern die langfristigen Kosten des Opportunismus den kurzfristigen Vorteilen überwiegen (Axelrod 1987).

${ }^{18}$ In Vorbereitung des anlaufenden Forschungsprojekts, auf dessen Basis die gestellten Forschungsfragen empirisch untersucht werden sollen, haben wir solche Treffen besucht.
} 
Allerdings muss auch eine Mindestgröße erreicht werden, um ein verschiedenartiges Angebot bereitzustellen.

\section{Marktalternative oder Marktergänzung?}

Vor allem die anhaltend hohe Arbeitslosigkeit, der zunehmende Anteil der Langzeitarbeitslosen und die damit verbundene steigende Armut regen in Deutschland die Diskussion um das Ende der Arbeitsgesellschaft und mögliche Alternativen an (Offe 1986; Gorz 1989; Beck 2000; Rifkin 2004). Laut Claus Offe und Rolf Heinze (1990, S. 17) wird im Zuge der Massenarbeitslosigkeit „die tendenziell 'wertlosere' Ressource Zeit immer reichlicher verfügbar [wird], der Zugriff auf die immer 'wohlfahrtsrelevantere' Ressource 'Geld', für wachsende Teile der Bevölkerung, zunehmend problematisch und prekär.“ In der Debatte um die individuellen und gesellschaftlichen Folgen der Arbeitslosigkeit finden sich Vorschläge zu neuen (oder zumindest in Vergessenheit geratenen) Quellen der Wohlfahrtsproduktion. Dabei lautet eine zentrale Frage, inwieweit die Abkehr von marktvermittelter Arbeit Potenziale zur Lösung individueller und gesellschaftlicher Problemlagen beinhaltet. Einschlägige Beiträge beschäftigen sich bevorzugt mit Themengebieten wie Eigenarbeit, bürgerschaftlichem Engagement und anderen informellen Tätigkeiten (Beck 2000; Jessen et. al. 1990; Maier 1985; Mutz 1997; Offe/Heinze 1986). Noch bevor das Konzept der Tauschringe in Deutschland Verbreitung fand, trafen Offe und Heinze bei ihrer Suche nach „sozialen 'Technologien““ (1990, S. 86), die brachliegende Fähigkeiten „’am Geldmedium vorbei'“ (1990, S. 86) nutzbar machen können, auf das Modell der ,Kooperations-’ oder ,Tauschringe’ und bewerteten es als richtungweisenden Ausweg aus der „Modernisierungsfalle“ ${ }^{\text {privater Haushalte. }}{ }^{19}$ Mit einem alternativen Markt für Güter und Dienstleistungen könnten die Selbstversorgungsfähigkeiten privater Haushalte qualitativ und quantitativ gesteigert und die Effizienznachteile aufgrund mangelnder Haushaltsgröße kompensiert werden. (Offe/Heinze 1986, S. 473; 491f; 1990, S. 77). Doch lassen sich diese Ansprüche auch erfüllen? Stellen die Tauschringe eine geeignete Alternative zur Versorgung über die reguläre Ökonomie dar oder sind sie lediglich eine Ergänzung zum Markt?

Unsere These lautet, dass Tauschringe reguläre Märkte nicht ersetzen können. Sie nehmen vielmehr die Rolle einer ergänzenden und kompensatorischen „Nebenökonomie“ im haushaltsnahen Wohnumfeld ein (Offe/Heinze 1990, S. 308). Insbesondere in wirtschaftlich

\footnotetext{
${ }^{19}$ Die Modernisierungsfalle besteht darin, dass im Zuge der „Verbetrieblichung der privaten Haushaltssphäre“ (Offe/Heinze 1986, S.473; S. 491) die kulturelle und motivationale Grundlage sowie das notwendige „Humankapital“ zur Selbstversorgung verloren gegangen sind. Erschwerend wirken auch die aus der geringen Größe moderner (häufig: Ein-Personen-) Haushalte resultierenden „diseconomies of small scale“ (Offe/Heinze 1986; S. 491).
} 
schwachen Regionen bei sozial benachteiligten Personen können sie dazu beitragen, den Lebensstandard anzuheben. Durch ihre Ausrichtung auf die Prinzipien der Subsidiarität und lokalen Autonomie kann es ihnen gelingen, die Abhängigkeit der Betroffenen von Geld abzuschwächen (Bowring 1998, S. 91; Pacione 1998, S. 70; Williams 1995, S. 329, Caldwell 2000; Meier 2001; North 1999). Der Angebotsschwerpunkt von Tauschringen liegt vor allem im Bereich von Dienstleistungen, wobei es sich hauptsächlich um „Jedermannstätigkeiten“ also „voraussetzungsarme“ Tätigkeiten - handelt (Offe/Heinze 1990; S. 309). Viele Tauschringteilnehmer gestalten ihr Angebots- und Nachfrageverhalten nach dem Motto: „Was ich gerne mache, biete ich an. Was ich dagegen nur sehr ungern erledige, frage ich bei einem Tauschpartner nach.“ Außerdem werden in den Austauschnetzen vorzugsweise Dienste organisiert, die in der formalen Ökonomie kaum angeboten werden, da sie entweder sehr spezifisch oder am Markt unverhältnismäßig teuer wären (Offe/Heinze 1990, S. 209). Meist handelt es sich um einfache Haushalts- und Renovierungshilfen wie beispielsweise Fensterputzen, Näharbeiten, Malerarbeiten oder Reparaturen. Die Tauschsysteme eröffnen denjenigen Personen, die aufgrund geringer monetärer Ressourcen ihre Bedürfnisse nicht über die regulären Märkte befriedigen können, die Möglichkeit, zumindest einige Dienstleistungen und Güter innerhalb dieser Alternativwirtschaft zu erhalten. ${ }^{20}$

Dabei spielt die Alternativwährung eine zentrale Rolle. Mit ihrer Hilfe soll die Abhängigkeit von der nationalen Währung, deren Verfügbarkeit in den meisten Fällen eng an Erwerbsarbeit gebunden ist, aufgebrochen werden (Pacione 1998, S. 65; Williams 1997). Sie kann durch die Beteiligung am „Tauschsystem“ mit am Arbeitsmarkt (vorübergehend oder dauerhaft) nicht gefragten Fähigkeiten verdient werden. Das lokale Geld stellt kein Wertaufbewahrungsmittel, sondern lediglich ein Mittel des Austausches dar (Pacione 1997, S. 417). Die Attraktivität von Tauschringen speist sich auch daraus, dass sie ohne Steuer- und Abgabenlast agieren. Dies ist sicherlich ein Faktor, der Einfluss auf die Preisgestaltung und damit auf die Anreize zur Teilnahme nimmt. In der Realität von Tauschringen ist das Aktivitätsniveau der Teilnehmer allerdings eher gering. Eine gerade angelaufene eigene empirische Erhebung zu Tauschringen dreier unterschiedlicher Regionen zeigt, dass der durchschnittliche monatliche Zeitaufwand für die Tauschgeschäfte bei gerade 4,1 Stunden liegt. ${ }^{21}$ Andere Studien des deutschsprachigen Raums zeigen eine ähnlich geringe Beteiligung (Meier 2001, S. 171). Dieses geringe Aktivitätsniveau spricht für sich: Steuerersparnisse innerhalb der Tauschringe sind nur marginal und die bisher bekannten Aktivitäten von Tauschringen scheinen die „Geringfügigkeitsgrenze“ nicht zu verletzen. Die ideologischen Motive, die sich

\footnotetext{
${ }^{20}$ Auch das andere Extrem ist denkbar: Personen können in den Tauschringen „Luxusdienste“ und „-güter“ nachfragen, die ihnen das allägliche Leben erleichtern (Pacione 1997, S. 421).

${ }^{21}$ Es handelt sich um eine Befragung von Teilnehmer/innen im Tauschring „LETS-München“ (N=182), bei drei Tauschringen aus dem Münchner Umland (N=190) und beim „Batzentauschring“ Leipzig (N=119). Zum Zeitpunkt dieses Artikels waren allerdings erst 83 Fragebögen aus der Region Leipzig erfasst.
} 
schon bei der Gründung der Tauschringe als bedeutsam erweisen, und Beweggründe der Gesellung, also etwa auf Gleichgesinnte zu treffen, sind offensichtlich für die Gestaltung der Tauschprozesse selbst ebenfalls nicht zu unterschätzen.

Trotz des wohl geringen Aktivitätsniveaus könnten die Möglichkeiten einer arbeitsmarkt- und sozialpolitischen Integration der Tauschringe ernsthaft bedacht werden. Denkbar wäre beispielsweise eine Teilnahme der Bezieher von Unterstützungsleistungen an dieser Art von Komplementärökonomie. Die nachgewiesene aktive Beteiligung an Tauschringen könnte von der Arbeitsagentur ähnlich wie die 1-Euro-Jobs als ein bestandener Test auf Arbeitsbereitschaft akzeptiert werden. Die Beteiligung an den Tauschaktivitäten würde nicht nur die niedrigen Einkommen in der wohlfahrtsstaatlichen Unterstützung erträglicher machen, sondern den betroffenen Personen selbst positive Entwicklungspotenziale bieten. Einigen Studien im englischsprachigen Raum zu Folge können die Teilnehmer an Tauschsystemen nicht nur ihren Lebensstandard verbessern, sondern sich durch ihre Aktivität in dieser informellen Ökonomie auch marktfähige Fertigkeiten bewahren oder neu hinzu erwerben. Innerhalb der Tauschringe können die Betroffenen Kontakte finden und pflegen, die bei der Suche nach Arbeitsplätzen wertvoll und gewinnbringend sein können. Tauschringe können also dabei helfen, die negativen Folgen der Arbeitslosigkeit zu lindern, und stellen für einige Personen ein „Sprungbrett“ in ein formales Beschäftigungsverhältnis dar (Williams 1996b; 1996c; 1997; 2001). Dieses Potenzial haben die Tauschringe in Deutschland momentan nicht. Daher mutet das angedeutete Szenario für Deutschland sehr optimistisch an. Um die positiven Chancen der Tauschsysteme in Deutschland nutzen zu können, müsste zunächst die Stellung der Tauschringe in der Arbeitsmarkt- und Sozialpolitik klar definiert und Transparenz bezüglich ihren Möglichkeiten geschaffen werden. Es müssten einfache Bewertungskriterien für im Tauschring erbrachte Leistungen eingeführt und die Geringfügigkeitsgrenzen für diese speziellen Arrangements angepasst werden. Da es sich bei einer Integration in die Arbeitsmarkt- und Sozialpolitik zwangsläufig nicht mehr ausschließlich um auf Freiwilligkeit basierende Zusammenschlüsse handelt, werden außerdem Kontroll- und Anreizsysteme notwendig, um den langfristigen Systemfortbestand zu gewährleisten. Lokale Austauschnetzwerke schneiden hinsichtlich der Verbesserung der Lebenslage von einkommensschwachen Haushalten und dem Zugang zum regulären Arbeitsmarkt in denjenigen Ländern deutlich besser ab, die sie in ihrer staatlichen Politik berücksichtigen wie beispielsweise Australien und Neuseeland (Fitzpatrick 2000; Liesch/Birch 2000; Williams 1996b). Die Integration der Tauschringe in die Arbeitsmarkt- und Sozialpolitik ist sicher ein ambitioniertes Vorhaben, dem sorgfältige Evaluationen und Konzeptionen vorangehen müssten. 


\section{Ausblick}

Das Phänomen der Tauschringe berührt verschiedene klassische soziologische Fragen. Zunächst veranschaulicht die Entstehung der Tauschringe die „Macht der Ideen“, wie sie seit Durkheim und Weber in der Soziologie thematisiert wird. Ohne ihren ideologischen Hintergrund wären soziale Bewegungen wie die Tauschringbewegung nicht möglich. Die Ideologien mobilisieren zur Teilnahme, sie sind Ausdruck der Interessen und Motive von politischen Entrepreneurs. Weiterhin verweist die Untersuchung von Tauschringen auf Prozesse der Institutionalisierung. Tauschringe sind auf Regeln angewiesen, wie etwa das Währungssystem oder die Eintrittsbedingungen, die wiederum selbst mehr oder weniger gut an die ideologischen Ziele angepasst sind. Institutionen besitzen eine normative und eine regulative Komponente (Scott 2001). So sind Tauschringe und ihre Regeln mit Werten und allgemeinen Normen verbunden, die den Bestand des Systems über ein rein ökonomisches Kalkül hinaus beeinflussen können. Hier ist beispielsweise an Vorstellungen von Fairness, Gemeinschaft oder Gesellung zu denken. Da es bei Regelverletzungen kaum Sanktionen und schon gar keine externe Durchsetzung der Sanktionen gibt, sind die Tauschringe auf die Entwicklung von Regulativen aus sich selbst heraus angewiesen. Zu erwarten ist, dass soziale Strukturen die notwendigen regulativen Elemente hervorbringen können. Während bei Tauschgeschäften im anonymen Markt keine Unterstützung durch soziale Strukturen bezogen werden kann, gewinnt diese Komponente gerade in begrenzten, personalisierten Umgebungen an Bedeutung. Denn der gegenseitige Umgang und die Tauschtransaktionen selbst werden in solchen Umgebungen erheblich erleichtert. Daher wird häufig versucht, Transaktionen, die ursprünglich auf dem Spot-Markt abgewickelt werden, in dauerhafte, wechselseitige Geschäftsbeziehungen umzuwandeln (Raub 1999). In begrenzten Umgebungen mit einer vergleichsweise geringen Zahl von Teilnehmern verlieren vertragliche Absicherungen zunehmend an Bedeutung dagegen fällt die soziale Struktur des Tauschprozesses mehr ins Gewicht. James Coleman (1988) hat dieses Phänomen an der Organisation des Diamantenhandels in New York veranschaulicht. Die engen Beziehungen der Händler untereinander ersetzen in diesem Kontext jede Diebstahlversicherung. Ähnliche Effekte gehen von den Tauschringen aus, denn obwohl monetäre Anreize und die Möglichkeit einer vertraglichen Absicherung ausgeschaltet sind, ist das Opportunismuspotenzial in diesen Systemen äußerst gering. Die Tauschbeziehungen sind temporal und strukturell „eingebettet“ - und die Art der Einbettung entscheidet mit über den langfristigen Bestand des Systems. Die Soziologie kann hier auch ihre wissenschaftliche Identität herausstellen: Lange Zeit wurden Austauschbeziehungen isoliert und aus ihrem sozialen Kontext losgelöst betrachtet. Am Beispiel der untersuchten lokalen Austauschnetzwerke, die sich ideologisch von jeglichen marktwirtschaftlich-monetären An- 
reizen zur Lösung der Kooperationsprobleme distanzieren, wird deutlich, dass diese unsoziologische Sichtweise zu kurz greift. Vertrauen ist ein wesentliches „Schmiermittel“ auch von wirtschaftlichen Austauschprozessen (Arrow 1974).

Schließlich liefert die Untersuchung von Tauschsystemen auch Beiträge zur aktuellen soziologischen „Zeitdiagnose“. Es geht um die Notwendigkeit und Tragfähigkeit eines informellen Sektors. Inwieweit sind hier nicht gegenläufige Prozesse zu beobachten? Ein Teil der Bevölkerung scheint aus Gründen zu geringer Einkommen verstärkt auf die Erbringung bzw. die Produktion und den Kauf von Dienstleistungen und Waren außerhalb des regulären Marktes angewiesen. Gleichzeitig existieren Bestrebungen, genau diese Art von haushaltsnahen Dienstleistungen in den allgemeinen Arbeitsmarkt zu integrieren. Interessant ist daher, ob mit einer Förderung des Niedriglohnsektors ein Rückgang der Tauschaktivität verbunden ist. Damit werden unmittelbar individuelle Teilnahmemotivationen betrachtet. Sind also doch prekäre Situationen am Arbeitsmarkt und nicht so sehr normative Zielsetzungen die treibende Kraft für eine Teilnahme an den Systemen? Das Szenario, wonach Teilnehmer aufgrund weiterer Beschäftigungsmöglichkeiten aus den Tauschringen ausscheiden, trägt zur Diskussion der in den Sozialwissenschaften weit verbreiteten, aber sehr umstrittenen Parallelitätshypothese bei. Diese geht davon aus, dass mit sinkendem Einkommen das Potenzial zu Tätigkeiten in der informellen Ökonomie abnimmt. Arbeitslosen gehen wesentliche soziale Netzwerkkontakte verloren, die über Betätigungsmöglichkeiten in der informellen Ökonomie informieren. Aufgrund ihrer Einkommensrestriktionen können sie sich außerdem die materiellen Voraussetzungen, also beispielsweise Transportmittel und Werkzeug, die für eine Beteiligung an der informellen Ökonomie erforderlich sind, nicht mehr leisten (Glatzer/Berger/Schmitt 1986; Jessen/Siebel 1988; Pahl 1984; Pahl/Wallace 1985; Renooy 1990). Die Varianz der existierenden Tauschringe bezüglich ihrer Aktivität ist jedenfalls ein wesentliches Erkenntnisziel weiterer Forschungsbemühungen. ${ }^{22}$ Es spricht einiges für spezifische Entwicklungspfade zu Tauschringen mit eher hohem und eher geringem Tauschvolumen.

\footnotetext{
${ }^{22}$ Wir werden diese Fragen in einem im April 2005 angelaufenen Forschungsprojekt (http://www.uni-konstanz.de/hinz/austausch/) auch empirisch untersuchen.
} 


\section{Literatur}

Abraham, Martin/Kropp, Per (2000): Die institutionelle und soziale Einbettung von Suchprozessen für wirtschaftliche Transaktionen: Das Beispiel der Wohnungssuche, in: Regina Metze/Kurt, Mühler/Karl-Dieter Opp (Hrsg.), Normen und Institutionen: Entstehung und Wirkungen. Leipzig: Leipziger Universitätsverlag, S. 415-431

Abraham, Martin/Voss, Thomas (1999): Das Zahlungsverhalten von Geschäftspartnern. Eine Untersuchung des Zahlungsverhaltens im Handwerk für den Raum Leipzig, Arbeitsbericht des Instituts für Soziologie Nr. 2. Leipzig

Aldrich, Howard E. (1999): Organizations Evolving, Thousand Oaks: Sage Publications

Aldrich, Howard E./Pfeffer, Jefrey (1976): Environments of Organization, in: Annual Review of Sociology 2, S. 79-105

Aldrich, Howard E./Auster, Ellen R. (1986): Even Dwarfs Stared Small, in: Research in Organizational Behavior 8, S. 165-198

Anheier, Helmut (2000): Movement Entrepreneurs and Organisational Development: The Role of "Single Members" in the German NAZI Party, 1925-30. Incomplete Draft, Center for Civil Societies, London School of Economics

Arrow, Kenneth J. (1974): The Limits of Organization, New York, London: W.W. Norton

Astley, Graham W. (1985): The Two Ecologies: Population and Community Perspectives on Organizational Evolution, in: Administrative Science Quarterly 30, S. 224-241

Axelrod, Robert (1987): Die Evolution der Kooperation, München: Oldenbourg

Baum, Joel A. (1996): Organizational Ecology, in: Stewart Clegg/Cynthia Hardy/Walter R. Nord (Hrsg.), Handbook of Organizational Studies, London: Sage Publications, S. 77-114

Batenburg, Ronald S./Raub, Werner/Snijders, Chris (2000): Vertrauen und Verträge: Eine empirischtheoretische Analyse der Effekte "sozialer Einbettung" auf die Steuerung wirtschaftlicher Transaktionen durch Normen und Institutionen, in: Regina Metze/Kurt Mühler/Karl-Dieter Opp (Hrsg.), Normen und Institutionen: Entstehung und Wirkungen, Leipzig: Leipziger Universitätsverlag, S. 85-413

Beck, Ulrich (2000): Wohin führt der Weg, der mit dem Ende der Vollbeschäftigungsgesellschaft beginnt? in: Ulrich Beck (Hrsg.), Die Zukunft von Arbeit und Demokratie, Frankfurt am Main: Suhrkamp, S. 7-66

Bebbington, Jahn (2000): Local Exchange Trading Systems (LETS): An Introduction and Evaluation of the Challenges to Accounting, Draft 14.5.2000

Binn, Felix G. (1982): ... die Gesell kennen, ohne ihn zu nennen und die ihn nennen, ohne ihn zu kennen, in: Zeitschrift für Sozialökonomie 53, S. 23-25

Bowring, Finn (1998): LETS: An Eco-Socialist Initiative? in: New Left Review 232, S.91-111

Brittain, Jack W./Freeman, John H. (1980): Organizational Proliferation and Density Dependent Selection, in: John R. Kimberly/Robert H. Miles, and Associates (Hrsg.), The Organizational Life Cycle, San Francisco: Jossey Bass Publishers, S. 291-338

Brüderl, Josef/Preisendörfer, Peter/Ziegler, Rolf (1992): Survival Chances of Newly Founded Business Organizations, in: American Sociological Review 57, S. 227-241

Caldwell, Caron (2000): Why do People Join Local Exchange Trading Systems? in: International Journal of Community Currency Research 4, http://www.le.ac.uk/ulmc/ijccr/vol4-6/4no1.htm (22.08.05)

Carmen, Raff (1997): LETS (Local Exchange Trading Systems): A Local "Win-Win” Counterpractice in a Global "Win-Lose" Economy, in: Development 40, S.75-79

Carroll, Glenn R. (1983): A Stochastic Model of Organizational Mortality: Review and Reanalysis, in: Social Science Research 12, S. 303-329

Coleman, James S. (1988): Social Capital in the Creation of Human Capital, in: American Journal of Sociology 94, S. 95-121

Coleman, James S. (1990): Foundations of Social Theory, Cambridge: Harvard University Press

Dobrev, Stanislav D./Tai-Young, Kim/Hannan, Michael T. (2001): Dynamics of Niche Width and Resource Partitioning, in: American Journal of Sociology, 106, S. 1299-1337 
Ekins, Paul (1986): The Living Economy: A New Economics in the Making, London: Routledge

Fichman, Michael/Levinthal, David (1991): Honeymoons And the Liability of Adolescence: A New Perspective on Duration Dependence in Social and Organizational Relationships, in: Academy of Management Review 16, S. 442-468

Fitzpatrick, Tony (2000): LETS and Benefit Claiming in the UK: Results of a Pilot Project, in: International Journal of Community Currency Research 4, http://www.le.ac.uk/ulmc/ijccr/vol4-6/4no6.htm (22.08.05)

Flap, Henk D. (1988): Conflict, Loyalty, and Violence, Frankfurt am Main: Lang

Freeman, James/Carroll, Glenn R./Hannan, Michael T. (1983): The Liability of Newness: Age Dependence in Organizational Death Rates, in: American Sociological Review 88, S. $692-710$

Glatzer, Wolfgang/Berger-Schmitt, Regina (1986): Haushaltsproduktion und Netzwerkhilfe. Die alltäglichen Leistungen der Familien und Haushalte, Frankfurt am Main: Campus

Godschalk, Hugo (1986): Die geldlose Wirtschaft. Vom Tempeltausch bis zum Barter-Club, Berlin: Basis

Gorz, André (1989): Kritik der ökonomischen Vernunft. Sinnfragen am Ende der Arbeitsgesellschaft, Berlin: Rotbuch

Gran, Even (1998): Green Domination in Norwegian LetSystems: Catalyst for Growth or Constraint on Development? in: International Journal of Community Currency Research 2, http://www.le.ac.uk/ulmc/ijccr/vol1-3/2no3.htm (22.08.05)

Granovetter, Mark S. (1985): Economic Action and Social Structure: The Problem of Embeddedness, in: American Journal of Sociology 91, S. 481-510

Granovetter, Mark S. (1990): The Old and the New Economic Sociology: A History and an Agenda, in: Roger Friedland/AF Robertson (Hrsg.), Beyond the Market Place: Rethinking Economy and Society, New York: Aldine de Gruyter S. 89-112

Haug, Sonja (2000): Vertrauen ist gut, Kontrolle ist besser? Soziales Kapital und moralische Normen im Kommunitarismus, in: Regina Metze/Kurt Mühler/Karl-Dieter Opp (Hrsg.), Normen und Institutionen: Entstehung und Wirkungen, Leipzig: Leipziger Universitätsverlag, S. 321-357

Hannan, Michael T./Carroll, Glenn R. (1992): Dynamics of Organizational Populations: Density, Legitimation and Competition, New York: Oxford University Press

Hannan, Michael T./Carroll, Glenn R. (1995): An Introduction to Organizational Ecology, in: Glenn R. Carroll/Michael T. Hannan (Hrsg.), Organizations in Industry, New York: Oxford University Press, S. 17 - 31

Hinz, Thomas (1998): Betriebsgründungen in Ostdeutschland, Berlin: Edition Sigma

Hoeben, Corine (2003): LETS be a Community. Community in Local Exchange Trading Systems, Groningen: ICS Dissertation Series

Hubert, Eva-Maria (2004): Tauschringe und Marktwirtschaft. Eine ökonomische Analyse lokaler Komplementärökonomien, Berlin: Duncker \& Humblot

Jessen, Johann/Siebel, Walter/Siebel-Rebell, Christa/Walther, Uwe-Jens/Weyrather, Irmgard (1990): Informelle Arbeit bei Industriearbeitern, in: Rolf G. Heinze/Claus Offe (Hrsg.), Formen der Eigenarbeit: Theorie, Empirie, Vorschläge, Opladen: Westdeutscher Verlag, S. 74 -86

Jessen, Johann/Siebel, Walter/Siebel-Rebell, Christa/Walther, Uwe-Jens/Weyrather, Irmgard (1988): Arbeit nach der Arbeit. Schattenwirtschaft, Wertewandel und Industriearbeit, Opladen: Westdeutscher Verlag

Kimberly, John R. (1980): Initiation, Innovation, and Institutionalization in the Creation Process, in: John R. Kimberly, Robert H. Miles, and Associates (Hrsg.), The Organizational Life Cycle, San Francisco: Jossey-Bass Publishers, S. 15-43

Kleine, Andreas (1995): Entscheidungstheoretische Aspekte der Principal-Agent-Theorie, Heidelberg: Physical-Verlag

Kuhn, Norbert (2002): Tauschringe - Möglichkeiten und Grenzen einer „geldlosen“ Wirtschaft, Marburg: Marburger Beiträge zum Genossenschaftswesen 39

Lang, Peter (1994): LETS Work: Rebuilding the Local Economy, Bristol, Grover Books

Langseth, Trond (1993): Green Dollars in New Zealand: Local Money for Sustainable Development, Master Thesis, Universität Oslo

Lee, Roger (1996): Moral Money? LETS and the Social Construction of Local Economic Geographies in Southeast England, in: Environment and Planning A 28, S.1377-1394 
Liesch, Peter W./Birch, Dawn (2000): Community-Based LETSystems in Australia: Localised Barter in a Sophisticated Western Economy, in: International Journal of Community Currency Research 4, http://www.le.ac.uk/ulmc/ijccr/vol4-6/4no2.htm (22.08.05)

Macaulay, Stewart (1963): Non-Contractual Relations in Business: A Preliminary Study, in: American Journal of Sociology 28, S. 55-67

MacNeil, Ian R. (1978): Contracts: Adjustments of Long-Term Economic Relations under Classical, Neoclassical, and Relational Law, in: Northwestern University Law Review 72/6, S. 854 905

Maier, Hans E. (1985): Selbsthilfe zwischen Markt und Staat, in: Rudolf Brun (Hrsg.), Erwerb und Eigenarbeit. Dualwirtschaft in der Diskussion, Frankfurt am Main: Fischer Taschenbuch Verlag, S. 167-179

March, James G./Simon, Herbert A. (1958): Organizations, New York: Wiley

McAdam, Dough/McCarthy, John D./Zald, Mayer, N. (1996): Introduction: Opportunities, Mobilizing Structures, and Framing Processes - Toward a Synthetic, Comparative Perspective on Social Movements, in: Dough McAdam/John D. McCarthy/Mayer N. Zald (Hrsg.), Comparative Perspectives on Social Movements. Political Opportunities, Mobilizing Structures, and Cultural Framings, Cambridge: Cambridge University Press, S. 1-20

Meier Daniela (2001): Tauschringe als besondere Bewertungssysteme in der Schattenwirtschaft. Eine theoretische und empirische Analyse, Berlin: Duncker \& Humblot

Miles, Robert H. (1980): Findings and Implications of Organizational Life Cycle Research: A Commencement, in: John R. Kimberly/Robert H. Miles, and Associates (Hrsg.), The Organizational Life Cycle, San Francisco: Jossey-Bass Publishers, S. 430-450

Mutz, Gerd (1997): Dynamische Arbeitslosigkeit und diskontinuierliche Erwerbsverläufe. Wie stehen die Chancen für eine zukünftige Tätigkeitsgesellschaft? in: Berliner Debatte INITIAL 8, S. 2335

Niemeyer, Verena (2002): Tauschringe in Deutschland, Diplomarbeit an der Wirtschaftswissenschaftlichen Fakultät der Universität Heidelberg

North, Peter (1999): Explorations in Heterotopia: Local Exchange Trading Schemes (LETS) and the Micropolitics of Money and Livelihood, in: Environment and Planning D: Society and Space17, S. 69-86

Offe, Claus (1986): Sozialstaat und Beschäftigungskrise - Probleme der Sicherung der sozialen Sicherung, in: Peter Alheit/Gerd Lobodd/Jörg Wollenberg (Hrsg.), Wie wir leben wollen. Krise der Arbeitsgesellschaft, Widerstand, Reform und Perspektiven, Hamburg: VBA, S. 29-44

Offe, Claus/Heinze, Rolf G. (1986): Am Arbeitsmarkt vorbei. Überlegungen zur Neubestimmung „haushaltlicher“ Wohlfahrtsproduktion in ihrem Verhältnis zu Markt und Staat, in: Leviathan 14, S. 471-495

Offe, Claus/Heinze, Rolf G. (1990): Organisierte Eigenarbeit. Das Modell Kooperationsring, Frankfurt am Main: Campus

Olson, Mancur (1968): Die Logik des kollektiven Handelns. Kollektivgüter und die Theorie der Gruppen, Tübingen: Mohr Siebeck

Onken, Werner (1983): Ein vergessenes Kapitel der Wirtschaftsgeschichte - Schwanenkirchen, Wörgl und andere Freigeldexperimente, in: Zeitschrift für Sozialökonomie 57-58, S.3-20

Pacione, Michael (1997): Local Exchange Trading Systems - A Rural Response to the Globalization of Capitalism? in: Journal of Rural Studies 13, S. 415-427

Pacione, Michael (1998): Toward a Community Economy - An Examination of Local Exchange Trading Systems in West Glasgow, in: Urban Geography 19, S. 211-231

Pahl, Raymond E./Wallace, Claire (1985): Arbeitsstrategien von Haushalten in Zeiten wirtschaftlicher Rezession, in: Jürgen Krämer/Rainer Neef (Hrsg.), Krise und Konflikte in der Großstadt im entwickelten Kapitalismus, Basel, Boston; Stuttgart, S. 333

Pahl, Raymond E. (1984): Divisions of Labour, Oxford: Blackwell

PaySys (1997): LETSysteme und Tauschringe: Ein Handbuch über Formen und Ausgestaltungsmöglichkeiten lokaler Verrechnungssysteme, Frankfurt am Main (Manuskript)

Pennings, Johannes M. (1980): Environmental Influences on the Creation Process, in: John R. Kimberly/Robert H. Miles, and Associates (Hrsg.), The Organizational Life Cycle, San Francisco: Jossey-Bass Publishers, S. 134-160 
Peterson, Kerstin (1990): Nebenwährung als Sozialvertrag: Kanadische Erfahrungen mit dem "Local Employment and Trading System” (LETS), in: Rolf G. Heinze/Claus Offe (Hrsg.), Formen der Eigenarbeit. Theorie, Empirie, Vorschläge, Opladen: Westdeutscher Verlag, S. 147-158

Picot, Arnold (1999): Organisation. Eine ökonomische Perspektive, Stuttgart: Schaeffer-Poeschel Verlag

Pieper, Niklas (2002): Die rechtliche Struktur bargeldloser Verrechnungssysteme unter besonderer Berücksichtigung von Barter-Clubs und LET-Systemen, Berlin: Weißensee Verlag

Rao, Hayagreeva (2002): Gründung von Organisationen und die Entstehung neuer organisatorischer Formen, in: Jutta Allmendinger/Thomas Hinz (Hrsg.), Organisationssoziologie. Sonderheft der Kölner Zeitschrift für Soziologie und Sozialpsychologie 42, S. 319-344

Raub, Werner (1999): Vertrauen in dauerhaften Zweierbeziehungen: Soziale Integration durch aufgeklärtes Eigeninteresse, in: Soziale Integration. Sonderheft 39, Kölner Zeitschrift für Soziologie und Sozialpsychologie, S. 239-268

Raub, Werner/Weesie, Jeroen (1990): Reputation and Efficiency in Social Interactions: An Example of Network Effects, in: American Journal of Sociology 96, S. 626 -654

Renooy, Piet (1990): The Informal Economy: Meaning, Measurement and Social Significance. Amsterdam: Netherlands Geographical Studies 115 (Regioplan)

Rifkin, Jeremy (2004): Das Ende der Arbeit und ihre Zukunft. Neue Konzepte für das 21. Jahrhundert, Frankfurt am Main: Campus

Romanelli, Elaine (1989): Organizational Birth and Population Variety: A Community Perspective on Origins, in: Research in Organizational Behavior 11, S. 211-246

Schneider, Friedrich/Enste, Dominik (2000): Schattenwirtschaft und Schwarzarbeit - Umfang, Ursachen, Wirkungen und wirtschaftspolitische Empfehlungen, München: Oldenbourg

Schneider, Christian (1995): Barter-Clubs. Chancen und Probleme. Eine theoretische und empirische Analyse, Berlin: Duncker \& Humblot

Schraven, Jorim (2000): The Economic of Local Exchange and Trading Systems: A Theoretical Perspective, in: International Journal of Community Currency Research 4, http://www.le.ac.uk/ulmc/ijccr/vol4-6/4no5.htm (22.08.05)

Schraven, Jorim (2001): Mutual Credit Systems and the Commons Problem: Why Community Currency Systems such as LETS need not Collapse under Opportunistic Behaviour, in: International Journal of Community Currency Research 5, http://www.le.ac.uk/ulmc/ijccr/vol4-6/5no4.htm (22.08.05)

Scott, Richard W. (2001): Institutions and Organizations, Thousand Oaks: Sage Publications (2.Auflage).

Seyfang, Gill (1997): Examining Local Currency Systems: A Social Audit Approach, in: International Journal of Community Currency Research 1, http://www.le.ac.uk/ulmc/ijccr/vol1-3/1no1.htm (22.08.05)

Seyfang, Gill (2002): Tackling Social Exclusion with Community Currencies: Learning From LETS To Time Banks, in: International Journal of Community Currency Research 6, http://www.le.ac.uk/ulmc/ijccr/vol4-6/6no3.htm (22.08.05)

Smelser, Neil/Swedberg, Richard (Hrsg) (2005): Handbook of Economic Sociology, Princeton: Princeton University Press (2. Auflage).

Stanley, Steven M. (1981): The New Evolutionary Timetable, New York, Basic Books

Stinchcombe, Arthur (1965): Social Structure and Organizations, in: James G. March (Hrsg.): Handbook of Organizations. New York: Rand McNally, S. 142-193

Snijders, Chris (1996): Trust and Commitments, Thesis Publishers: Amsterdam

Täubner, Mischa (2002): Vom Stamme Nimm und Gib, in: Die Zeit 28

Thorne, L (1996): Local Exchange Trading Systems in the United Kingdom: A Case of ReEmbedding? in: Environment and Planning 28, S. 1361-1376

Unterguggenberger, Silvio (1983): 50 Jahre Wörgler Freigeld, in: Zeitschrift für Sozialökonomie 59, S. 37-41

Voss, Thomas (1998): Vertrauen in modernen Gesellschaften. Eine spieltheoretische Analyse, in: Regina Metze/Kurt Mühler/Karl-Dieter Opp (Hrsg.), Der Transformationsprozess. Analysen und Befunde aus dem Leipziger Institut für Soziologie, Leipzig: Leipziger Universitätsverlag, S. 91-129

Weber, Max (1965): Wirtschaft und Gesellschaft, Tübingen: Mohr Siebeck 
Whittier, Nancy E. (1997): Political Generations, Micro-Cohorts, and the Transformation of Social Movements, in: American Sociological Review 62, S. 760-778

Williams, Colin C. (1995): The Emergence of Local Currencies, in: Town and Country Planning 64/12, S. 329-332

Williams, Colin C. (1996a): Local Exchange and Trading Systems: A New Source of Work and Credit for the Poor and Unemployed?, in: Environment and Planning A 28, S. 1395-1415

Williams, Colin C. (1996b): The New Barter Economy: An Appraisal of Local Exchange and Trading Systems (LETS), in: Journal of Public Politics 16, S. 85-101

Williams, Colin C. (1996c): Informal Sector Responses to Unemployment: An Evaluation of the Potential of Local Exchange Trading Systems (LETS), in: Work, Employment \& Society 10, S. 341-359

Williams, Colin C. (1997): LETS in Australia: A New Tool for Community Development, in: International Journal of Community Currency Research 1, http://www.le.ac.uk/ulmc/ijccr//vol1-3/1no3.htm (22.08.05)

Williams, Colin C. (1998): Evaluating LETS as a Means of Tackling Social Exclusion and Cohesion, in: International Journal of Community Currency Research 2, http://www.le.ac.uk/ulmc/ijccr/vol1-3/2esrc.htm (22.08.05)

Williams, Colin C.; et al. (2001): The Role of the Third Sector in Paving a 'Third Way': Some Lessons from Local Exchange and Trading Schemes (LETS) in the United Kingdom, in: International Journal of community currency research 5 , http://www.le.ac.uk/ulmc/ijccr/vol4-6/5no3.htm (22.08.05)

Williamson, Oliver (1985): The Economic Institutions of Capitalism, New York: Free Press

Zelizer, Viviana (1994): The Social Meaning of Money, New York: Basic Books 